\title{
Giant Solitary Hydatid Cyst of the Spleen - A Rare Presentation
}

\author{
Authors \\ Dr Nisha Attri, Dr Sushma Kanojiya, Dr Madan Solanki \\ J.N.U.I.M.S.R.C \\ Email: nisha.attri4@gmail.com, Mobile: 9819458676
}

\section{Introduction}

Hydatid cyst is a zoonotic disease more prevalent in endemic areas. The disease is caused by the larval stage of a tapeworm, Echinococcous granulosus. The primary hosts are, usually dogs and the intermediate hosts are sheep and cattle. Humans are incidental host in the dog-sheep life cycle of Echinococcus granulosus. The hydatid cysts grow upto $5-10 \mathrm{~cm}$ in size and can be asymptomatic for years or even decades ${ }^{(1)}$. Mostly it is an incidental finding detected by imaging studies $^{(2)}$. Symptoms are usually due to the space occupying effect of the cystic lesion or complications such as rupture of the cyst ${ }^{(3)}$. Liver and lungs are the commonly affected organs although it can involve any organ ${ }^{(1)}$. Primary infestation of the spleen by the parasite is a rare event and splenic involvement represents less than $2 \%-5 \%$ of all cases of human hydatid disease reported $^{(3,4)}$. Splenic involvement may be direct or indirect by the arterial route, once the parasite passes through the liver and lungs. Splenic hydatid cysts are usually solitary. We report on a thirty-year-old female with primary splenic hydatid cyst, which is a rare event.

Keywords: Solitary, splenic cyst, hydatid cyst, Echinococcus granulosus, histopathology.

\section{Case Presentation}

A 30 year old female, not a known case of any chronic illness, presented with dragging pain and swelling in the left hypochondriac region Family history was taken and it was discovered that she had been living in a rural area, was shepherd by profession. Her abdominal physical examination revealed enlargement of spleen, with a mild tenderness at the left outer quadrant of the abdomen. She had no other abdominal complaints. Her laboratory investigations, including complete blood picture, urine routine and microscopic examination, blood electrolytes, liver functions tests were within normal limits. Abdominopelvic ultrasound showed a cystic mass with a smooth margin in the spleen measuring $13 \mathrm{~cm} \mathrm{x} 12 \mathrm{~cm} \mathrm{x}$ $11 \mathrm{~cm}$. (Figure 1). There was no evidence of any cystic lesion in the other parts of the abdomen such as the liver and peritoneum. The chest X-ray did not show any cysts. Our diagnosis was a splenic hydatid cyst based on imaging findings. Splenectomy was performed and the specimen was received for histopathological assessment. Grossly the spleen was enlarged. A cyst measuring approximately $13 \mathrm{~cm}$ in diameter was present. On cut section, a unilocular cyst was seen filled with clear fluid along with granular sand like deposits. The cyst wall was whitish and 0.1- 


\section{JMSCR Vol||07||Issue||02||Page 294-297||February}

$0.2 \mathrm{~cm}$ in thickness (Figure 2) The splenic parenchyma was significantly reduced due to pressure atrophy. The histopathological examination confirmed the diagnosis of splenic hydatid cyst.

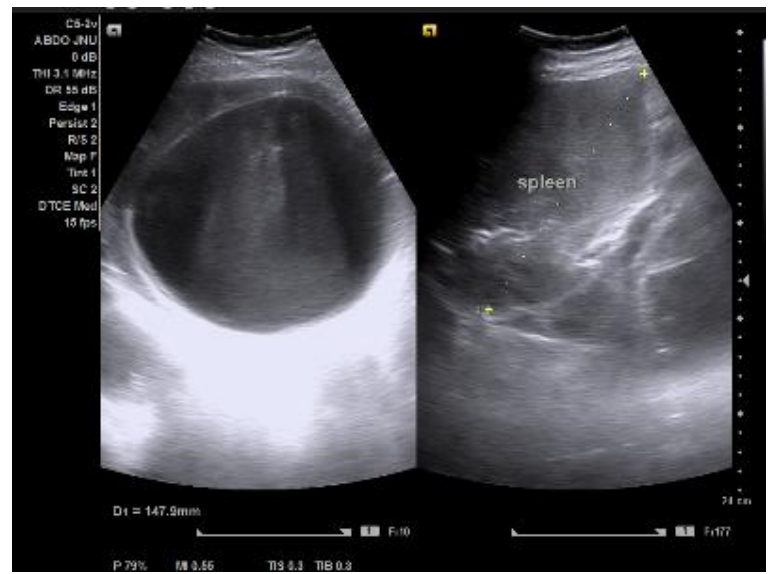

Figure 1: USG Abdomen- A large cystic lesion in splenic parenchyma

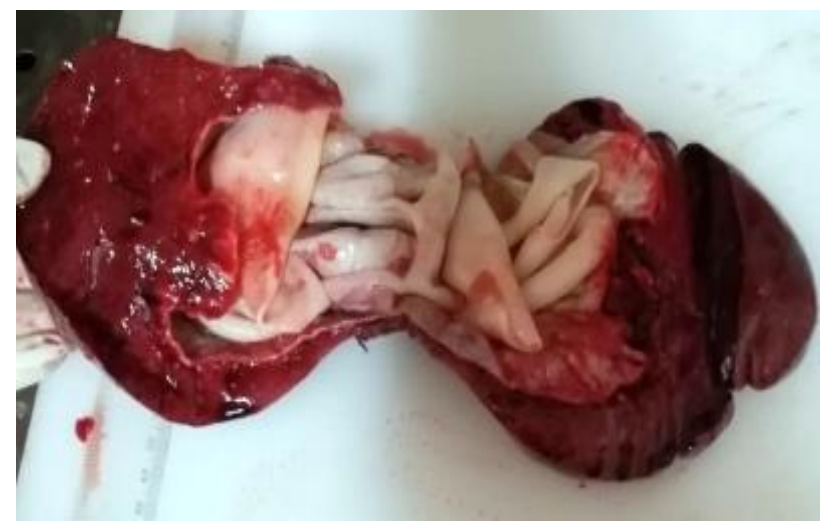

Figure 2: Gross - An unilocular hydatid cyst. The cyst wall was whitish and 0.1-0.2 cm in thickness. The splenic parenchyma was significantly reduced due to pressure atrophy.

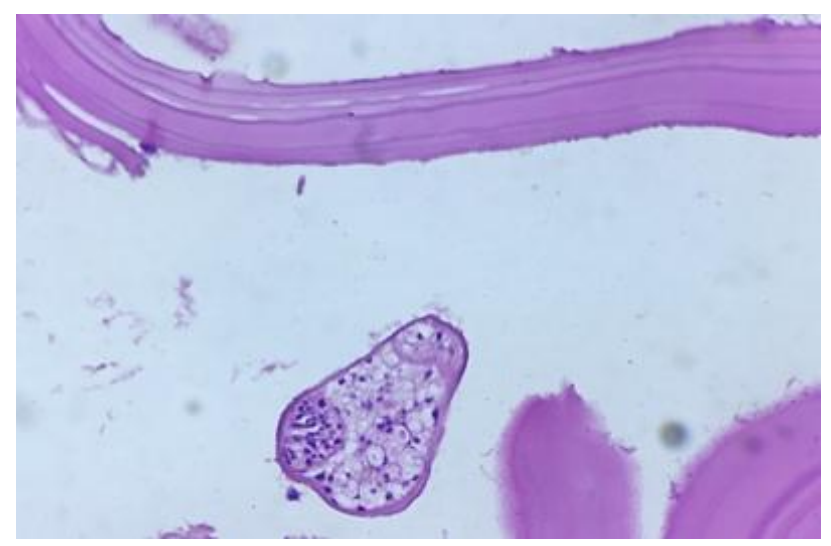

Figure 3: Microscopy- Scolex, acellular chitinous lamellated wall and germinal membrane.(10X)

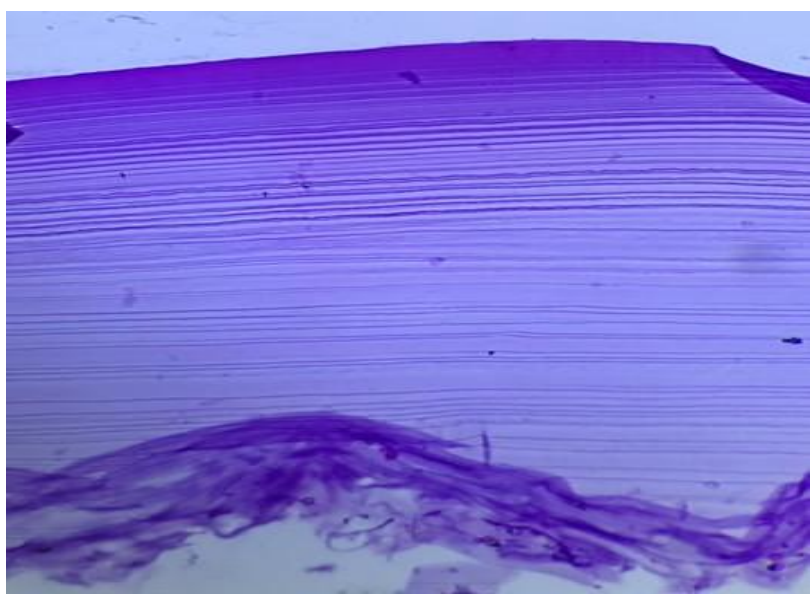

Figure 4: Microscopy- PAS stain positive acellular lamellar cyst wall.(40x)

\section{Discussion}

Hydatid disease, a worldwide zoonosis, is prevalent in most sheep raising countries in Asia, Australia, South America, Eastern and Southern Europe $^{(1)}$. Two classes of parasites are mainly involved, Cestodes (flat worms, tape worms) and Nematodes (round worms). Echinococcus granulosus is a tapeworm and its adult form lives in the intestinal tract of infected dogs. Its eggs are excreted in the dog's faeces and swallowed by intermediate hosts such as sheep, cattle or humans. Humans get infected either by direct contact with a dog or by ingestion of food contaminated by the eggs. After ingestion of the eggs, larvae penetrates the mucosa of the jejunum reaching, via the venous and lymphatic channels, to every organ of the body ${ }^{(1,5)}$. Hydatid cyst may develop in any part of the body. The location is commonly hepatic $(75 \%)$ then pulmonary $(15 \%)$ and only $10 \%$ occur in the rest of the body. Splenic hydatid cyst is rare and the incidence is reported between $2 \%-3 \%$ and $5 \%$ by different authors. Splenic involvement of hydatid cyst has a reported frequency of $0.5 \%-6 \%$ with abdominal hydatidosis ${ }^{(4,6)}$. Primary infestation of the spleen usually takes place by the arterial route after the parasite has passed the two filters, hepatic and pulmonary. A retrograde venous route, which bypasses the lung and liver, has also been reported. Secondary splenic hydatid cyst usually follows intra peritoneal spread or systemic dissemination following ruptured hepatic hydatid 
cyst. Splenic hydatid cysts are usually asymptomatic but may present as a painful mass in the left upper guardant. The complications of splenic hydatid cyst are mainly infection, intraabdominal rupture and fistulization to the bowel, mainly colon. Rupture of the cyst leading to spleno thoracic fistula has also been reported. Hydatid disease can involve various organs like the brain, bones, genital system, bowels, subcutaneous tissue, peritoneal cavity, the heart and soft tissues. In children the lung is the most common site of involvement. Hydatid cysts with unusual localization may cause serious problems in the differential diagnosis ${ }^{(4,6)}$. Anaphylactic reactions due to rupture of the cyst have been reported leading to fever; in addition dyspnea has also been described with splenic involvement ${ }^{(5,7)}$. Hydatid cysts may be single or multiple, uniloculated or multiloculated. Specific signs include visualization of calcification of the cyst wall, the presence of daughter cyst, and membranes detachment. The main differential diagnosis of splenic hydatidosis is splenic cystic lesions such as pseudocyst, hematoma, abscess and cystic neoplasms. Biochemical and hemagglutination tests may be helpful and eosinophilia may be the finding on hematological investigation $^{(1,3)}$.

The definitive diagnosis is based on abdominal ultrasound, CT scan and histopathological examination which have greater sensitivity and specificity ${ }^{[8]}$. On ultrasound of abdomen, splenic hydatid cyst may present as a solitary unilocular or rarely multilocular well defined anechoic spherical cystic lesions or may demonstrate an anechoic spherical cystic lesion with hyperechoic marginal calcification as seen in this case. CT abdomen confirms the cystic lesion with or without daughter cysts within the spleen with attenuation value near that of water and does not enhance after intravenous contrast administration. It is more sensitive than ultra sonography in depicting subtle wall calcification.

On histopathological examination, the hydatid cyst consists of 3 layers: adventitia formed of compressed host tissue, middle layer of friable ectocyst and an inner germinal layer from which large number of scolices are produced, which are the heads of developing worms. Daughter cysts are formed if the viability is threatened. Histopathologically our case was also characterized by scolices, an acellular lamellar cyst wall and germinal membrane. (Figure 3,4). Different serological tests can also be carried out for the diagnosis. These include the hydatid immunoelectrophoresis, enzyme linked immunoabsorbent assay (ELISA), latex agglutination and indirect hemagglutination test .

\section{Conclusion}

Although echinococcosis is found most often in liver and lungs, it seems that any organ can be involved by this zoonotic parasitic disease. The clinical manifestations of hydatid disease depend on location, size, and stage of development of cysts. Hydatid disease is largely asymptomatic until complications occur due to unusual localizations that may cause serious problems of diagnostic confusion. Hydatid disease must be considered in the differential diagnosis of cystic lesions, especially in patients who have spent time in endemic areas. The histopathologic findings of scolices, acellular lamellated cyst wall and germinal membrane are diagnostic. The combination of clinical history, radiological , histopathological findings and serological test results are valuable in diagnosing atypical Hydatid disease. Surgical removal and use of protoscolicidal agents are the most effective treatments of hydatid cysts. This disease can rarely cause substantial morbidity and mortality.

\section{References}

1. Polat P, Kantarci M, Alper F, Suma S, Koruyucu MB, Okur A. Hydatid disease from head to toe. Radiographics. 2003;23(2):475-94.

2. Safioleas M, Misiakos E, Manti C. Surgical treatment for splenic hydatidosis. World J Surg. 1997;21(4):374-7. 
3. Fernandez-Ruiz M, Guerra-Vales JM, Enguita-Valls AB, Vila-Santos J, GarciaBorda FJ, Morales-Gutierrez C. Splenic hydatid cyst, a rare location of extrahepatic echinococcosis: Report of six cases. Eur J Intern Med. 2008;19(7):e51-3.

4. Schlittler LA, Dallagasperina VW. Nonparasitic splenic cysts. Rev Col Bras Cir. 2010;37(6):442-6.

5. Ahmadi NA, Badi F. Human hydatidosis in Tehran, Iran: a retrospective epidemiological study of surgical cases between 1999 and 2009 at two university medical centers. Trop Biomed. 2011;28(2):450-6.

6. Pukar MM, Pukar SM. Giant solitary hydatid cyst of spleen-A case report. Int J Surg Case Rep. 2013;4(4):435-7.

7. Culafic DM, Kerkez MD, Mijac DD, Lekic NS, Rankovic VI, Lekic DD, et al. Spleen cystic echinococcosis: clinical manifestations and treatment. Scand J Gastroenterol. 2010;45(2):186-90.

8. Herrera Merino N, Abascal Morte J, Diaz del Rio Botas $\mathrm{M}$ et al. Splenic hydatid cyst. A report of 16 cases (Article in Spanish). Ev Esp Enferm Dig 1991;79:254-8. 